\title{
ATYPICAL HAEMOLYTIC UREMIC SYNDROME
}

\section{Immunohaematology}

Dr Somasekar R Professor, Department of Pediatrics, Sree Balaji Medical College \& Hospital, Chennai.

\section{ABSTRACT}

This is a case of 7-year-old child who presented with typical characteristics of HUS who developed complications in various systems but responded with timely treatment.

\section{KEYWORDS}

\section{INTRODUCTION}

Haemolytic-uremic syndrome (HUS) is a, life threatening condition presenting with thrombotic microangiopathy with a triad Microangiopathic haemolytic anaemia (nonimmune, Coombsnegative)Thrombocytopenia Acute kidney injury.

This syndrome occurs following an infection with Shigelladysenteriae and Escherichia coli which has the ability to produce Shiga-like toxins in $90 \%$ paediatric population

Atypical HUS is not related to Shiga toxins seen in $10 \%$ cases of all HUS. There is high mortality, risk of recurrences and permanent renal damage in atypical HUS.

A case of Atypical Haemolytic Uremic Syndrome is reported because of rare occurrence and high morbidly associated with the condition.

\section{CASE REPORT}

A 9-year-old boy presented

High grade fever for 4-5days

Facial puffiness

Reduced urine output

Passing brown coloured urine for 2 days

Petechial rashes, skin peeling, abdominal pain for 1 day; restlessness and irritability for 1 day.

No history of any diarrhoea or blood in stools.

He had a history of gaining $2 \mathrm{~kg}$ weight and progressively increasing pallor in the last 1 week.

He also had a past history of sore throat, fever, generalized rashes and strawberry tongue 4 weeks prior to presentation; for which he was been treated with antibiotics in OPD.

On Examination

Severe pallor

Facial puffiness (started from periorbital region)

Generalized scaling and petechial rashes, all over the body.

Vitals HR - 100/min RR - 26/min; BP - 160/110 mmHg; SpO2 - 97\% in room air

Systemic examination showed hepatomegaly $(3 \mathrm{~cm}$ BCM); rest of the examination was normal.

\section{Investigations}

Complete blood count: Suggestive of acute intravascular haemolysis $\mathrm{P} / \mathrm{S}$ showing fragmented RBCs, helmet cells; schistocytes; haemoglobinuria; very low serum haptoglobin Normal reticulocyte count Mild hyperbilirubinemia (serum bilirubin : $2.9 / 0.6 \mathrm{mg} / \mathrm{dl}$ )

DCT negative direct Coomb's Test thrombocytopenia (platelet counts : 28000/cu mm)

Acute renal failure (oliguria; azotemia; hypertension and fluid retention).

Blood urea and serum creatinine levels at admission were $123 \mathrm{mg} / \mathrm{dl}$ and $1.8 \mathrm{mg} / \mathrm{dl}$ respectively serum lactate dehydrogenase (LDH) levels: 1894U/L (normal: $150-450 \mathrm{U} / \mathrm{L}$ )

ASO level was normal and autoimmune workup was WNL.
A presumptive diagnosis of atypical haemolytic uremic syndrome a HUS was considered.

Factor $\mathrm{H}$ antigen (CFH) level was $199 \mathrm{mg} / \mathrm{h}$ (normal $170-270 \mathrm{mg} / \mathrm{h}$ ); C3 was $49-9 \mathrm{mg}(\mathrm{dl})$ [normal $79-152 \mathrm{mg}(\mathrm{dl})]$;

C4 level was 35-8 mg(dl) [normal 16-38 mg(dl)]

\section{Treatment \& Course of Illness:}

\section{Hypertension}

Nitro-glycerine infusion (for initial 3 days) along with multiple oral anti-hypertensive drugs (atenolol, amlodipine).

Acute renal failure 3 cycles haemodialysis on alternate days were done.

Due to high suspicion of atypical HUS .He was started on Plasmapheresis on day 3 of admission .

A total of 21 cycles of Plasmapheresis were done

Initially daily for 11 days followed by alternate day for 10 days later twice weekly for another 2 weeks and then once a week; under continuous monitoring of haemoglobin levels, platelet counts and LDH levels.

On day 6 of admission, the child developed multiple seizures along with altered sensorium and tonic posturing which were controlled with 2 anticonvulsants (phenytoin, levetericetam).

MRI brain was done which revealed multiple cerebral infarcts suggestive of acute ischemic insult.

He was started on hypertonic saline and dexamethasone.

He deteriorated over next 24 hours; sensorium worsened (GCS - E3 M4 V2) and developed left sided hemiparesis.

Child had features of raised ICT along with unequal pupils and was electively intubated and ventilated. Gradually, he developed righ sided weakness; repeat MRI brain showed new infarcts on the left side. He was started on LMW heparin and intravenous immunoglobulin (IVIG $2 \mathrm{gm} / \mathrm{kg}$ ).

His thrombophilia workup was normal.

Was started on Inj. Rituximab (Monoclonal antibodies); child showed definate improvement; his antibodies to complement factor $\mathrm{H}$ were repeated after 18 cycles of Plasmapheresis which decreased to $308 \mathrm{AU} / \mathrm{ml}$

He was extubated after 3 days and was discharged after 45 days of hospital stay in a conscious state with slurred speech and residual left sided gaze palsy and $\mathrm{B} / \mathrm{L}$ residual neurological deficit; nasogastric feeding and multiple antihypertensive medications were continued. His renal functions completely recovered.

His anti CFH ab levels done 2 months after discharge were $151 \mathrm{AU} / \mathrm{ml}$ and after 5 months post discharge were $59 \mathrm{AU} / \mathrm{ml}$

\section{DISCUSSION}

This is a sporadic case of atypical HUS and fulfilled all criteria for a severe form of this disease.

During his illness, the child experienced a number of complications 
such as septicaemia, refractory hypertension, and central nervous system involvement with residual sequel.

Haemolytic-uremic syndrome (HUS) is classified into 2 main categories:

Typical HUS (Shiga-like toxin associated HUS) is the classic, primary or epidemic form of haemolytic-uremic syndrome (HUS).

It is largely a disease of children younger than 2-3 years and often results in diarrhoea (formerly known as D+HUS).

Although Shiga like toxin-producing E. coli-HUS (STEC-HUS) strains most often triggered HUS, certain Shiga toxin-secreting strains of S. dysenteriae can also cause HUS

Acute renal failure occurs in $55-70 \%$ of patients, but they have a favorable prognosis and as many as $70-85 \%$ of patients show recovery of renal function.

Atypical HUS (non-shiga associated HUS [non-Stx-HUS])

$10 \%$ of all HUS cases and can be sporadic or familial.

Although STEC-HUS is relatively common in children, aHUS occurs in individuals of all ages

There is a poor prognosis in the first aHUS attack and associated with a mortality rate of $25 \%$, and out $50 \%$ of cases develop end-stage renal disease requiring dialysis and/or, irreversible brain damage (formerly known as D-HUS).

The familial form is associated with genetic abnormalities of the complement regulatory proteins. STEC-HUS is characterized by diarrhoea accompanied by bloody stools.

However, diarrhoea may also be present in some aHUS cases wherein it could be a manifestation of ischemic colitis. Also, enteritis that is not caused by STEC can trigger aHUS.

Therefore, a diagnosis of STEC-HUS cannot be made based on symptoms alone, and the earlier nomenclature of "D+HUS" and "DHUS" is not used at present.

STEC HUS should be confirmed by stool culture, the direct detection of shiga toxins, or the detection of anti-lipopolysaccharide-IgM antibodies.

The leading risk factors of atypical HUS are, however, genetic and acquired disorders of complement regulatory pathways which causes the pathological activation of the complement system, though no complement related abnormality is noted in approximately $40 \%$ of patients with aHUS.

Various triggers have been identified: non enteric infections, viruses, drugs, malignancies, transplantation, other underlying medical conditions like systemic lupus erythematosus.

Streptococcus pneumoniae infection accounts for $40 \%$ of all causes of atypical HUS and $4.7 \%$ of all causes of haemolytic-uremic syndrome (HUS) in children in the United States.

The disease is usually severe; causes respiratory distress, neurologic involvement, and coma, with a mortality rate of up to $50 \%$.

Familial Atypical HUS accounts for $<3 \%$ of all cases of HUS

Both autosomal dominant and autosomal recessive forms of inheritance are observed.

Genetic abnormalities in the complement regulatory proteins, including $\mathrm{C} 3$, factor $\mathrm{H}$, factor $\mathrm{B}$, factor $\mathrm{I}$, and $\mathrm{CD} 46$ (membrane cofactor protein, $\mathrm{MCP}$ ) are implicated.

Factor H: Factor H (HF1) consists of 20 homologous units called short consensus repeats (CSRs) and plays an important role in the regulation of the alternative pathway of complement and it protects glomerular endothelial cells and the basement membrane against complement attack by binding to the polyanionic proteoglycans on the surface of endothelial cells and in the sub endothelial region.
Clinical presentation includes prodrome of fever, irritability, lethargy, seizures ,acute renal failure, hypertension, edema, fluid overload and pallor.

Laboratory studies include kidney function tests, complete blood picture including peripheral smear for hemolysis, coagulation profile, D-dimer, $\mathrm{LDH}$, serum haptoglobin, urine routine examination, urine for hemoglobin and Ultrasound KUB.

\section{Clinical practice guidelines}

IN atypical HUS published in United Kingdom recommends extensive investigations of the status of complement system in patients with D-HUS including expression levels of complement factors in serum,expression of complement regulators on cell surfaces, genetic analysis and autoantibody screening.

Abnormalities in complement regulation can be associated with decreased $\mathrm{C} 3$ levels, and in most cases a normal C4 levels.

Also plasma and serum concentration of the terminal complement complex C5b-C9 should be estimated.

An increased plasma and serum level of C5b-C9 suggests increased complement activation in atypical HUS. Plasma levels of complement regulators complement factor $\mathrm{H}, \mathrm{B}$ and I should be measured though a normal level of these proteins does not eliminate the possibility of mutations in the genes that encode them (CFH $30 \%$ and CFI $10 \%$ of atypical HUS).

Mutations in CD46 gene is seen in 10\% of patients with D-HUS

The presence of autoantibodies against CFH (anti FH antibodies) is another relevant cause of complement dysregulation and should be assessed in all patients of suspected D-HUS. Since not all mutations associated with atypical HUS affect the secretion of the mutant protein, genetic analysis of $\mathrm{CFH}, \mathrm{CD} 46, \mathrm{CFI}, \mathrm{CFB}$ and $\mathrm{C} 3$ is mandatory for all patients.

\section{Guidelines for the diagnosis of aHUS}

Definitive diagnosis of aHUS is made when the triad of

Microangiopathic haemolytic anaemia

Thrombocytopenia

$\mathrm{AKI}$ is present.

The disease should have no association with Shiga toxins, and Thrombotic thrombocytopenic purpura (TTP) should also be excluded.

TTP is another form of thrombotic microangiopathy (TMA) caused by a deficiency of ADAMTS13 (a dis integrin and metalloproteinase with a thrombospondin type 1 motif, member 13); diagnosed based on the classic pentad (microangiopathic haemolytic anaemia, thrombocytopenia, labile psychoneurotic disorder, fever, and renal failure). However, the discovery of ADAMTS13 led to the finding that $60-90 \%$ of patients with TTP have a marked reduction in the activity of ADAMTS13, to a level of $<5 \%$. Therefore, patients with markedly reduced levels of ADAMTS13 activity $(<5 \%)$ favour TTP, thereby ruling out a diagnosis of aHUS.

\section{Probable diagnosis}

A probable diagnosis of aHUS is made when 2 of the following 3 conditions are found: microangiopathic haemolytic anaemia, thrombocytopenia, and AKI. The disease should have no association with Shiga toxins and TTP should be excluded.

Definitions of microangiopathic haemolytic anaemia, thrombocytopenia, and AKI that have been established by the joint committee of the JSN/ JPS

A probable diagnosis of aHUS is made when 2 of the following 3 conditions are found: microangiopathic hemolytic anaemia, thrombocytopenia, and AKI. The disease should have no association with Shiga toxins and TTP should be excluded.

Cases where aHUS should be strongly suspected: If there are features that are characteristic of HUS, aHUS should be strongly suspected if the following criteria are fulfilled, regardless of the presence of diarrhoea: the patient is younger than 6 months of age; time of onset is unclear (latent onset); the patient has a history of HUS 
(recurrent case); history of anaemia of unknown cause; recurrent HUS after kidney transplantation; family history of HUS (excluding cases of food poisoning); the patient has no diarrhoea of bloody stools

\section{Treatment of Atypical HUS}

Treatment guidelines based on strong clinical evidence of efficacy are absent. At present, limited treatment options are available and the current first line options are:

- Plasma exchange is the initial treatment of choice in all patients with Atypical HUS and should be considered as early as possible in the disease course. The findings of unexplained thrombocytopenia and microangiopathic hemolytic anemia are sufficient to consider thrombotic microangiopathy and initiate plasma exchange. Plasma exchange removes potentially toxic substances from the circulation.Patients with abnormalities of soluble complement factors (CFH, CFI and CFB) seem to benefit more than those with CD46 deficiency. Plasma treatment should be started within 24 hours of the patient's presentation to decrease treatment failures and then continued once or twice a day for at least 2 days after complete remission.

- Kidney Transplantation has not been recommended for patients with $\mathrm{CFH}$ and $\mathrm{CFI}$ mutations as the recurrence rates are very high $(90 \%)$. The prognosis is better for patients with CD46 mutations. Anti CFH antibody titers should be minimized by plasma exchange before transplantation to prevent their possible deleterious effect on the graft. Since CFH and CFI are synthesized in the liver, isolated liver transplantation and combined liverkidney transplantation are other therapeutic options available for patients with mutations in $\mathrm{CFH}$ or $\mathrm{CFI}$ or for those with anti $\mathrm{CFH}$ antibodies.

- Monoclonal antibodies: This is a new therapeutic approach for treatment of aHUS. Ecluzimab is a humanized monoclonal antibody that blocks the cleavage of $\mathrm{C} 5$ and impedes the generation of potent anaphylatoxin $\mathrm{C} 5 \mathrm{a}$ and the formation of $\mathrm{C} 5 \mathrm{~b}-$ C9. It can be considered as a good treatment option for patients with atypical HUS associated with defects in both soluble and membrane bound complement regulators. The existing data on patients with atypical HUS in children is quite promising. Several reports of the successful use of monoclonal antibodies in patients of atypical HUS regardless of identified mutations or other risk factors, suggests that chronic Ecluzimab / rituximab therapy might provide meaningful clinical benefit as measured by the resolution of thrombotic microangiopathy. Our own experience has also been encouraging.

Supportive therapy includes maintenance of fluid and electrolyte balance, adequate blood-pressure control, prophylactic antiepileptic in patients with neurologic symptoms, control of azotemia, optimize nutrition and monitor renal function and hemodynamics.

Complications include renal failure, stroke, coma, seizures and bleeding complications

\section{Prognosis}

- Patients collectively have a poor prognosis, and as many as $5060 \%$ progress to ESRD ( $50 \%$ in those with the sporadic forms and $60 \%$ in those with the familial forms) or develop irreversible brain damage. About $25 \%$ die during the acute phase.

- The recurrence rate in patients receiving renal transplants is as high as $50 \%$, with graft loss occurring in more than $90 \%$ who have recurrence. Recurrence rates are higher in patients with HF1 mutation

\section{CONCLUSION}

Atypical haemolytic uremic syndrome (aHUS) is at thrombotic microangiopathy; occurs rarely; and comprises of triad of microangiopathic haemolytic anaemia, thrombocytopenia, and acute kidney injury. Recent studies show abnormalities in the mechanisms complement regulation as causes of aHUS. The prognosis is very poor, with the first aHUS attack being associated with a mortality rate of approximately $25 \%$, and with approximately $50 \%$ of cases resulting in end-stage renal disease requiring renal replacement therapy.

This case highlights a uncommon presentation of a rare disease which needs a very high index of suspicion keeping in mind the very high morbidity and mortality associated with it.

\section{REFERENCES}

1. Alejandra Rosales, Magdalena Riedl and Lothar B. Zimmerhackl. Atypical HUS : current diagnostic and therapeutic approaches. Nephrology nature reviews. 2010 volume 6.

2. Blackall DP, Marques MB. Hemolytic uremic syndrome revisited: Shiga toxin, factor $\mathrm{H}$, and fibrin generation. Am J ClinPathol. 2004;121 suppl:S81-8.

3. Burns ER, Lou Y, Pathak A. Morphologic diagnosis of thrombotic thrombocytopenic purpura. Am J Hematol. 2004;75(1):18-21.

4. Caprioli J, Bettinaglio P, Zipfel PF, et al. The molecular basis of familial hemolytic uremic syndrome: mutation analysis of factor $\mathrm{H}$ gene reveals a hot spot in shor consensus repeat 20. J Am SocNephrol, 2001;12(2):297-307.

5. Davis AK, Makar RS, Stowell CP, Kuter DJ, Dzik WH. ADAMTS13 binds to CD36: potential mechanism for platelet and endothelial localization of ADAMTS13. Transfusion. 2009;49(2):206-13.

6. Edey MM, Mead PA, Saunders RE, et al. Association of a factor $\mathrm{H}$ mutation with hemolytic uremic syndrome following a diarrheal illness. Am J Kidney Dis. 2008;51(3):487-90

7. Eremina V, Jefferson JA, Kowalewska J, et al. VEGF inhibition and renal thrombotic microangiopathy. NEngl J Med. 13 2008;358(11):1129-3.

8. Frank C, Werber D, Cramer JP, et al. Epidemic profile of Shiga-toxin-producing Escherichia coli O104:H4 outbreak in Germany NEngl J Med 2011:365(19):1771-80.

9. Fremeaux-Bacchi V, Kemp EJ, Goodship JA, Dragon-Durey MA, Strain L, Loirat C, et al. The development of atypical haemolytic-uraemic syndrome is influenced by susceptibility factors in factor $\mathrm{H}$ and membrane cofactor protein: evidence from two independent cohorts. J Med Genet. 2005;42(11):852-6.

10. Furlan M, Robles R, Galbusera M, et al. von Willebrand factor-cleaving protease in thrombotic thrombocytopenic purpura and the hemolytic-uremic syndrome. N Engl J Med. 1998;339(22):1578-84

11. George JN. ADAMTS13, thrombotic thrombocytopenic purpura, and hemolytic uremic syndrome. CurrHematol Rep. 2005;4(3):167-9.

12. Haspel RL, Jarolím P. The "cutting" edge: von Willebrand factor-cleaving protease activity in thrombotic microangiopathies. TransfusApher Sci. 2005;32(2):177-8.

13. Kojouri K, Vesely SK, George JN. Quinine-associated thrombotic thrombocytopenic purpura-hemolytic uremic syndrome: frequency, clinical features, and long-term outcomes. Ann Intern Med. 2001;135(12):1047-51

14. Lapeyraque AL, Wagner E, Phan V, et al. Efficacy of plasma therapy in atypical hemolyticuremic syndrome with complement factor $\mathrm{H}$ mutations. PediatrNephrol. 2008;23(8): 13636

15. McGannon CM, Fuller CA, Weiss AA. Different classes of antibiotics differentially influence Shiga toxin production. Antimicrob Agents Chemother. 2010;54(9):37903798.

16. Pediatrics International and Clinical and Experimental Nephrology by the Japan PediatricSociety and the Japanese Society of Nephrology. 2013.

17. Siegler RL. Management of hemolytic-uremic syndrome. J Pediatr. 1988;112(6): 101420

18. Siegler R, Oakes R. Hemolytic uremic syndrome; pathogenesis, treatment, and outcome. CurrOpinPediatr. 2005;17(2):200-4

19. Tarr PI, Gordon CA, Chandler WL. Shiga-toxin-producing Escherichia coli and haemolyticuraemic syndrome. Lancet. 19-25 2005;365(9464):1073-86.

20. Tsai HM, Lian EC. Antibodies to von Willebrand factor-cleaving protease in acute thrombotic thrombocytopenic purpura. N Engl J Med. 1998;339(22):1585-94.

21. Wong CS, Jelacic S, Habeeb RL, Watkins SL, Tarr PI. The risk of the hemolytic-uremic syndrome after antibiotic treatment of Escherichia coli O157:H7 infections. N Engl J Med. 2000;342(26):1930-6. 